Check for updates

Cite this: RSC Adv., 2019, 9, 19523

Received 8th April 2019

Accepted 17th June 2019

DOI: 10.1039/c9ra02628b

rsc.li/rsc-advances

\section{Tetracycline hydrochloride loaded citric acid functionalized chitosan hydrogel for wound healing}

\begin{abstract}
Hao Chen, $\dagger^{a}$ Biyun Li, $\dagger^{b}$ Bei Feng, ${ }^{c}$ Hao Wang, ${ }^{a}$ Huihua Yuan (DD ${ }^{* b}$ and Zhiwei $X u^{* a}$
Citric modified chitosan (CC) hydrogel containing antibacterial drugs is developed by the freezing and thawing treatment method. The SEM image of the CC hydrogel revealed a porous structure. The rheological properties, porosity, swelling rate, water uptake, tensile properties and in vitro degradation were found to be tunable via CC concentration. To enhance antibacterial properties, tetracycline hydrochloride $(\mathrm{TH})$ representing the drug model, was integrated into the $\mathrm{CC}$ hydrogel. The cumulative release of drug was also tunable via CC concentration. The drug loaded CC hydrogel showed enhanced antimicrobial activity against E. coli and S. aureus. In animal tests, it was found the TH loaded CC hydrogel accelerated the healing of the wounds created on rats. These results suggest that the drug loaded CC hydrogel has a promising future in wound healing as a wound dressing.
\end{abstract}

\section{Introduction}

An ideal wound dressing should protect the wound from bacterial infection, provide a moist wound healing environment, absorb the exudates from the wound surface, allow gas exchange, be removed easily without trauma to the wound, and also be biocompatible. ${ }^{1,2}$ Hydrogels, three-dimensional crosslinked hydrophilic polymers with a very high intrinsic content of water, promote moist healing, are non-adherent and cool the surface of the wound, which may lead to a marked reduction in pain; they therefore have high patient acceptability and are suitable as wound dressings for wound healing..$^{3-7}$ Currently, many different materials such as poly(vinyl alcohol) (PVA), poly(ethylene glycol) (PEG), polyvinylpyrrolidone (PVP), poly(methacrylates) (PMMA), carboxymethyl cellulose (CMC) and alginate have been used as hydrogel dressing materials. ${ }^{1,8-11}$ Chitosan is considered to be one of the most widely used nature biopolymer for biomedical applications, especially in the field of wound dressings due to its favorable biological activities such as hemostasis, biocompatibility, biodegradability and antimicrobial properties. ${ }^{12-15}$ However, some weaknesses, such as poor mechanical properties and low absorption in the physiological fluid, seriously hinder its biomedical applications. ${ }^{11}$ Blending of

\footnotetext{
${ }^{a}$ Department of Cardiothoracic Surgery, Shanghai Children's Medical Center, School of Medicine, Shanghai Jiao Tong University, Shanghai 200127, China. E-mail: xuzhiwei@scmc.com.cn

${ }^{b}$ School of Life Sciences, Nantong University, Nantong, Jiangsu 226019, China. E-mail: yuanhh@ntu.edu.cn

'Shanghai Pediatric Congenital Heart Disease Institute, Shanghai Children's Medical Center, School of Medicine, Shanghai Jiao Tong University, Shanghai 200127, China

$\dagger$ These authors contributed equally.
}

natural/synthetic polymers with chitosan has gradually become an important approach to improve the performance of chitosanbased materials. ${ }^{13,17,18}$ For instance, blending of poly(vinyl alcohol) with chitosan hydrogels can significantly improve their mechanical and absorbent properties in physiological fluid. ${ }^{16,19,20}$ Compared with blended approach, crosslinked method is much easier to form a homogenous structure with improved physicochemical properties. However, such crosslinkers as glutaraldehyde, formaldehyde are frequently used in the fabrication of chitosan based hydrogels, which are toxic to cell and tissue. ${ }^{21,22}$ Thus, biocompatible crosslinking agents are highly preferable for preparation of chitosan based hydrogels used as wound dressing. Citric acid, a ternary organic carboxylic acid, has been used as a biocompatible cross-linker of chitosan for various tissue engineering applications..$^{23,24}$ Recently, our group demonstrated the ionotropic cross-linking of chitosan hydrogel with citric acid for enhanced mechanical properties and chondrogenic differentiation of human mesenchymal stems cells in vitro. ${ }^{25}$ Notably, citric acid could provide the antimicrobial activity for wound dressing. ${ }^{26}$ To this end, it's valuable to evaluate the wound healing effect of citric-acid modified chitosan as a wound dressing.

One of the major problems facing the healing process is the bacterial infection, raising the urgent and substantial need for the incorporation of an antimicrobial agent in the wound dressings to prevent infection at the wound site and to enhance the wound healing efficiency. ${ }^{13,27}$ To the best of our knowledge, there was no study on the fabrication of antibiotics loaded citric-acid modified chitosan (CC) hydrogels as a wound dressing. The main objective of the present work is to develop an antimicrobial drug loaded chitosan hydrogel wound dressing. The surface morphology, properties, water absorption 
velocity, tensile properties and in vitro degradation of the CC hydrogels were evaluated. In addition, tetracycline hydrochloride $(\mathrm{TH})$ was used as a model drug to evaluate the drug release behavior and the anti-bactericidal properties of the drug-loaded CC hydrogel. Finally, the drug-loaded CC hydrogel were applied to full-thickness skin wounds in rats to demonstrate the feasibility of the hydrogel for wound healing.

\section{Experimental section}

\subsection{Materials}

Chitosan (deacetylation degree $\geq 95 \%$ ) was supplied by Aladdin (Shanghai, China). Acetic acid, citric acid and sodium hydroxide $(\mathrm{NaOH})$ were all purchased from Sinopharm Chemical Reagent (Shanghai, China). Lysozyme (MW = $14.4 \mathrm{kDa}, 6800 \mathrm{IU} \mathrm{mg}^{-1}$ ) were obtained from Sigma (USA). Tetracycline hydrochloride was supplied by Titan (Shanghai, China). All the chemicals were used without further purification.

\subsection{Preparation of the CC hydrogels}

CC hydrogels were prepared according to previous study. ${ }^{25}$ Briefly, different amounts of chitosan were dissolved in $3 \%(\mathrm{w} / \mathrm{v})$ acetic acid solution to the final concentrations of 1,2 , and $3 \mathrm{wt} \%$. Then, $0.3 \mathrm{wt} \%$ citric acid was then added to above solutions. Thereafter, a $10 \mathrm{~mL}$ of each CC solution was poured into a template and frozen at $-20{ }^{\circ} \mathrm{C}$ for $12 \mathrm{~h}$. The gels were thawed in $0.5 \mathrm{M} \mathrm{NaOH}$ solutions at room temperature and washed with deionized water until pH became neutral (Fig. 1).

\subsection{Characterization of the CC hydrogels}

The morphology of CC hydrogels was tested using scanning electron microscope (SEM, Gemini SEM 300) at an accelerating voltage of $15 \mathrm{kV}$, and the pore size of CC hydrogels was determined from analysis of the SEM micrographs of five different areas using ImageJ software. The porosity $(\varepsilon)$ of the CC hydrogels was determined according to the following equation: $\varepsilon=(1$ $\left.-\rho / \rho_{0}\right) \times 100 \%$, where $\rho$ is the bulk chitosan density, and $\rho_{0}$ is the density of the $\mathrm{CC}$ the $\mathrm{CC}$ hydrogels.

A Modular Compact Rheometer (MCR102) equipped with a cylinder mould was used to examine the rheological properties of CC solution. The samples were evaluated in the steady shear mode with the frequency ranging from 10 to $100 \mathrm{rad} \mathrm{s}^{-1}$ at $25{ }^{\circ} \mathrm{C}$.

CC hydrogel ( $1 \mathrm{wt} \%, 2 \mathrm{wt} \%$, and $3 \mathrm{wt} \%$ ) specimens of $3 \mathrm{~mm}$ thickness and $15 \mathrm{~mm}$ diameter were frozen at $-20{ }^{\circ} \mathrm{C}$ and freeze-dried. Dried samples were incubated at $37{ }^{\circ} \mathrm{C}$ in phosphate buffered saline (PBS, pH 7.4) for $4 \mathrm{~h}$. Swelling ratios of specimens in PBS was quantified by the formula of $\left(W_{\mathrm{e}}-W_{0}\right) /$ $W_{0}$, where $W_{\mathrm{e}}$ and $W_{0}$ represent the wet and dry weights, respectively.

The water absorption velocity of CC hydrogel ( $1 \mathrm{wt} \%, 2 \mathrm{wt} \%$, and $3 \mathrm{wt} \%)$ is given by the formula of $\left(W_{t}-W_{\mathrm{t} 0}\right) / W_{\mathrm{e}} \times 100 \%$, where $W_{t}$ is weight at 1, 1.5, 2 and $2.5 \mathrm{~min}$, respectively. $W_{\mathrm{to}}$ is initial weight of hydrogel with being wrung out water. Each group of samples was measured with triplicate.

Tensile tests proceeded using a tabletop uniaxial material testing machine (H5K-S, Hounsfield, United Kingdom) equipped with a $10 \mathrm{~N}$ load cell. Rectangular-shaped specimens $(30 \mathrm{~mm} \times 10 \mathrm{~mm} \times 5 \mathrm{~mm})$ were stretched at a constant crosshead speed of $0.5 \mathrm{~mm} \mathrm{~min}^{-1}$ until the specimen fractured. These hydrogels were all in the wet state when tested. At least 5 samples were tested for each type sample.

CC hydrogels (2.0 mL solution) were prepared as described above. The degradation of CC hydrogels was tested according previous study. ${ }^{28}$ Briefly, the gel was added to PBS ( $\mathrm{pH} 7.4$ ) containing $4 \mathrm{mg} \mathrm{mL}^{-1}$ of lysozyme and gently shaken at $37^{\circ} \mathrm{C}$. After appropriate intervals, the wet weight of the gel was measured. The lysozyme solution was exchanged every $24 \mathrm{~h}$. The degree of degradation (DD) was determined by the weight change equation: $\mathrm{DD}=\left(W_{1}-W_{2}\right) / W_{1} \times 100 \%$, where $\mathrm{DD}$ is the degree of degradation, $W_{1}$ is the initial weight of sample and $W_{2}$ is the final dry weight of the sample.
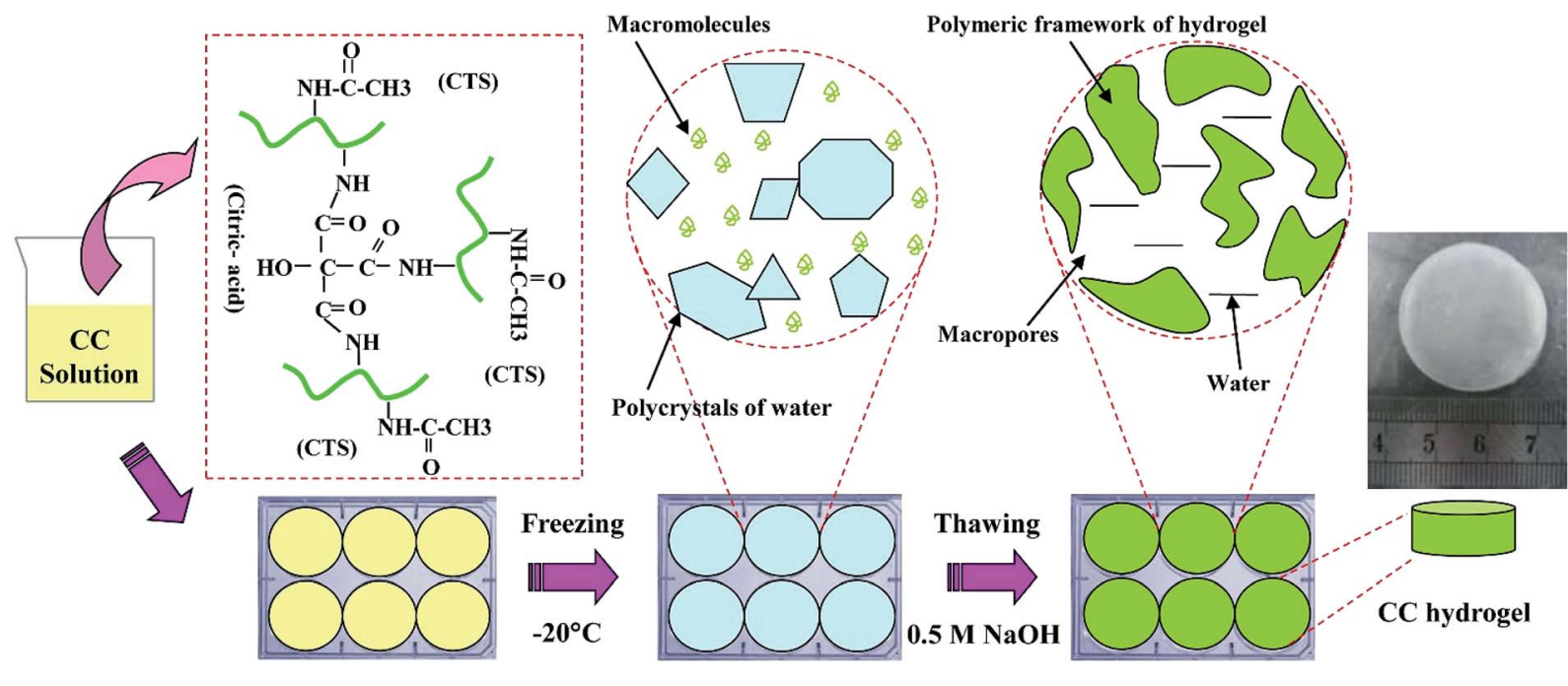

Fig. 1 Schematic for the preparation of CC hydrogel. 


\subsection{Drug loading and release studies}

Tetracycline hydrochloride (TH) was used as a model antibacterial drug to analyze the antibiotic loading and in vitro release of the CC hydrogel. To load TH in the CC hydrogels, $0.6 \mathrm{~mL}$ of TH solution ( $3 \mathrm{mg} \mathrm{mL}^{-1}$ ) poured on dried CC hydrogels $(10 \mathrm{mg})$ and then freeze-drying. In vitro release of antibacterial drug from the CC hydrogels was carried out in $10 \mathrm{~mL}$ phosphatebuffered saline (PBS, pH 7.4) with shaking at $37^{\circ} \mathrm{C} .5 \mathrm{~mL}$ of aliquot was taken and replaced with fresh PBS at specific time periods of $0,1,3,5,7,12$ and $24 \mathrm{~h}$. The amount of drug released in the aliquots was determined by measuring the absorbance of the solution at $370 \mathrm{~nm}$ for $\mathrm{TH}$ using an Evolution $300 \mathrm{UV}$ Spectrophotometer (Thermo).

\subsection{Assay of antibacterial activity}

The antimicrobial activity of CC was investigated against E. coli and $S$. aureus, which were precultured at $37{ }^{\circ} \mathrm{C}$ to reach a concentration of about $10^{8}$ colony forming unit per $\mathrm{mL}$ (CFU $\left.\mathrm{mL}^{-1}\right)$. All the test samples were cut into circular discs $(15 \mathrm{~mm}$ in diameter) for use. The zone of inhibition tests was adopted to evaluate the antimicrobial activity. The control (CC) and drug loaded samples (CC-TH) were placed on the E. coli or $S$. aureus growth agar plate and incubated at $37^{\circ} \mathrm{C}$ for $24 \mathrm{~h}$. The inhibition zone was calculated by measuring the diameter of the nearest whole millimeter of the inhibited growth around the sample disk, and was performed in triplicates.

\subsection{Animal test}

As The wound healing efficacies of the TH-CC wound dressings were evaluated using a rat model. All the experimental procedures were performed in strict accordance with the guidelines for the care and use of laboratory animals of Shanghai Jiao Tong University School of Medicine and also approved by Animal Care and Experiment Committee of Shanghai Jiao Tong University School of Medicine. The dorsal skin of male SD rats (6 weeks old) was shaved and disinfected using 75\% (v/v) ethanol under anesthesia with pentobarbital. Full-thickness skin wounds $3 \mathrm{~cm}$ in diameter were created by excising the dorsum of each animal and disinfected using $75 \%(\mathrm{v} / \mathrm{v})$ ethanol. A cylindrical TH-CC or CC hydrogels and dry cotton gauze were applied to each wound. After applying the dressing materials, the rats were housed individually in cages. Measurement of the wound area was performed every other day.

To perform histological analysis, skin tissues from the wound sites at 4, 8, and 12 days of post-wounding were retrieved and fixed in Bouin's solution for 7 days, and then washed by flowing water for $24 \mathrm{~h}$. After being bisected into pieces with a thickness of $2 \mathrm{~mm}$, all tissues were initially dehydrated in a graded series of alcohol and then embedded in paraffin. The transverse sections (4-5 mm thick) were prepared using rotatory microtome and stained with hematoxylin-eosin dye for histological evaluation.

\subsection{Statistical analysis}

All values were showed as the mean \pm standard error of at least three samples. Statistical analysis was performed using Origin 8.0 software with one way analysis of variance (ANOVA) of Tukey's test to determine differences between groups. A value of $P<0.05$ was considered to be statistically significant.

\section{Results and discussion}

\subsection{Rheological properties and microstructure analysis}

To analyse the rheological properties of different concentration of CC solution, the curves of storage modulus $\left(G^{\prime}\right)$ and loss modulus $\left(G^{\prime \prime}\right)$ of these solutions over frequency were recorded. Rheological data indicated that increasing CC solution concentration leads to increased elasticity behaviour (Fig. 2A). This is likely because of more entanglement and less relaxation of the CC molecular chains in higher concentration.

As expected, CC hydrogels were successfully obtained by freeze-thawing (Fig. 1). Surface morphology of different CC hydrogel with the varying $\mathrm{CC}$ concentration was evaluated using SEM as illustrated in Fig. 2B-D. All the CC hydrogels exhibit a similar pattern of pore morphology. The SEM image Fig. $2 \mathrm{C}$ is the reference and has been presented in our earlier study. ${ }^{25}$ The micropores structure is favourable for mass transport and nutrient supply. ${ }^{\mathbf{1 3 2 8}}$ The porosity of CC hydrogels, decreased from $90.5 \pm 0.9 \%, 89.2 \pm 1.3 \%$, to $86.1 \pm 1.8 \%$ as CC concentration increased from 1,2 , and $3 \mathrm{wt} \%$ respectively, while the pore wall thickness increased from $16 \pm 7 \mu \mathrm{m}, 19 \pm 8 \mu \mathrm{m}$, to 26 $\pm 11 \mu \mathrm{m}$, as the CC concentration increased from 1,2 , and $3 \mathrm{wt} \%$, respectively. The thicker wall leads to a higher mechanical strength as well as improved structural integrity.

\subsection{Swelling behaviour and water absorption capacity}

Swelling behaviour of hydrogels in PBS was investigated to evaluate their capacity to absorb wound exudation fluid. Fig. 3A shows the equilibrium swelling ratios of CC hydrogels $(1 \mathrm{wt} \%$, $2 \mathrm{wt} \%$, and $3 \mathrm{wt} \%$ ) in PBS. Swelling ratio decreased from 433.86 $\pm 15.09 \%$ ( $1 \mathrm{wt} \%$ ) to $386.98 \pm 14.03 \%$ for $3 \mathrm{wt} \%$. CC hydrogels with higher concentration have smaller swelling ratio, which validates the earlier speculation that greater wall thickness helps retain the structural integrity of the scaffold under wet conditions. In addition, an ideal wound dressing should be able to rapidly absorb the excessive secreted exudates from the wound and provide an optimal moisture environment for wound repair. ${ }^{30}$ In this work, CC hydrogels could absorb water quickly and swell up to $90 \%$ of their capacity within $1 \mathrm{~min}$, suggesting that it is sufficient to absorb the exudates for a period of time (Fig. 3B).

\subsection{Mechanical property}

For skin tissue engineering, hydrogels with sufficient mechanical strength that maintain structural integrity is necessary for skin regeneration at the site of implementation. ${ }^{29,31}$ Tensile tests were performed on each type of CC hydrogels to obtain the stress-strain relation from which modulus and strength were evaluated. The mechanical properties of the scaffolds improved 
A
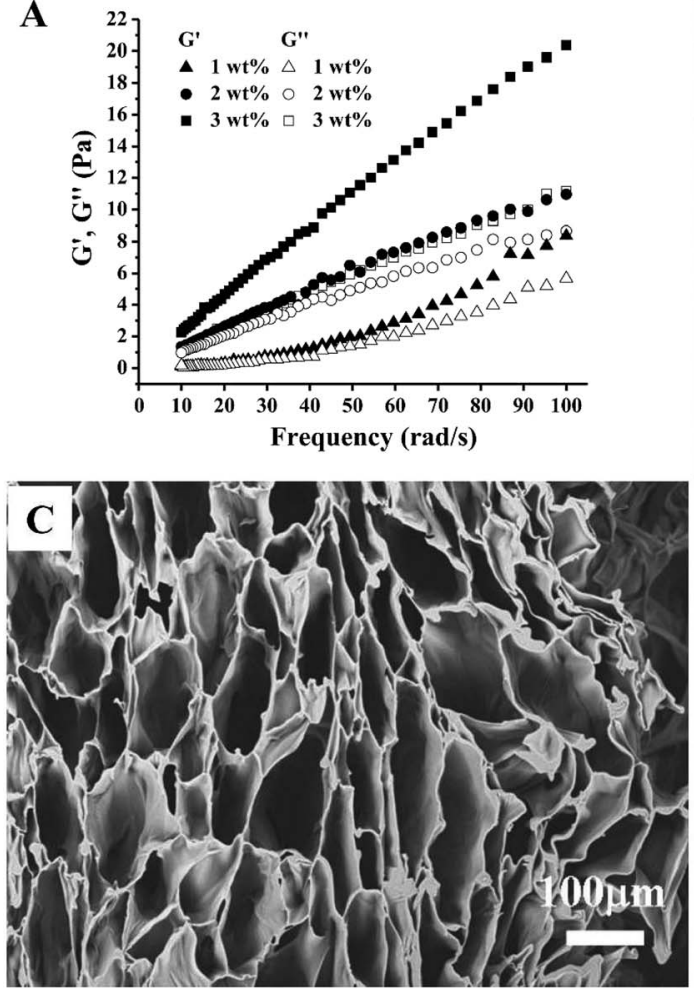
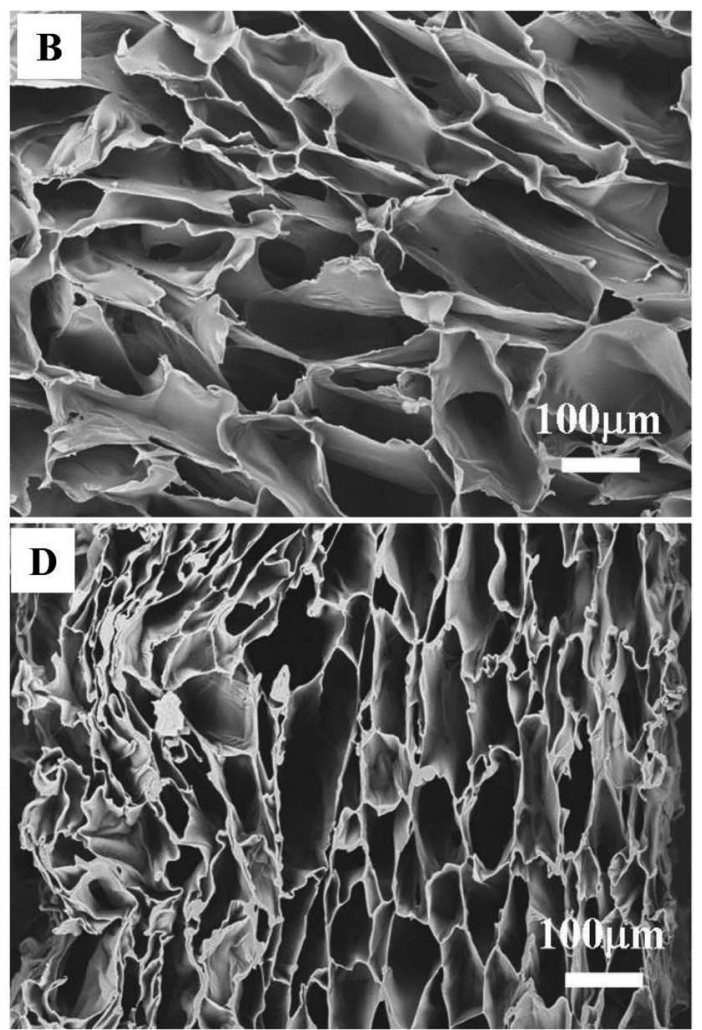

Fig. 2 (A) Rheological behavior of the CC solutions with different concentrations represented by storage modulus ( $G^{\prime}$, filled symbols) and loss modulus ( $G^{\prime \prime}$, unfilled symbols). SEM images of the $1 \mathrm{wt} \%$ (B), $2 \mathrm{wt} \%$ (C), and $3 \mathrm{wt} \%$ (D) CC hydrogels.

with increasing CC concentration (Fig. 4). Tensile strength and modulus increased from $1.79 \pm 0.41 \mathrm{MPa}$ and $15.67 \pm 2.44 \mathrm{MPa}$ (1 wt\%), respectively, to $2.01 \pm 0.01 \mathrm{MPa}$ and $16.12 \pm 0.11 \mathrm{MPa}$ (3 wt\%), which is higher than that of native skin (tensile strength: $3 \pm 1.5 \mathrm{MPa}$ (ref. 32) and modulus: $2.16 \mathrm{kPa}$ to $0.1 \mathrm{MPa}$ (ref. 33 and 34)). The above results provide us with a comprehensive understanding of CC hydrogels, which hold promise for serving as scaffold materials with tunable mechanical properties for soft skin tissue engineering, higher than that of CTS hydrogels $(0.05 \pm 0.02 \mathrm{MPa})$ (Fig. 4B).

\subsection{In vitro degradation}

Ideal tissue engineering scaffolds need to be degraded in a controlled manner and allow the restoration of the target tissue to its natural structure, morphology and function without leaving any foreign material behind. Chitosan is readily degraded by enzymes via the cleavage of the glycosidic bonds, particularly lysozyme, which is naturally present in various parts of the human body and tissues..$^{35}$ Thus, knowledge concerning the dependence of the degradation rate of the CC hydrogels by lysozyme is very important for the application of the gel to wound care. $1 \mathrm{wt} \% \mathrm{CC}$ hydrogels completely degraded within $54 \mathrm{~h}$ (Fig. 5). A higher concentration resulted in a longer

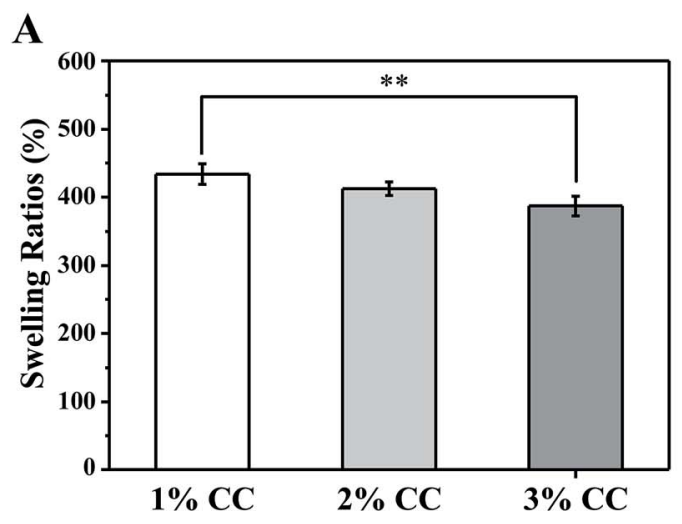

B

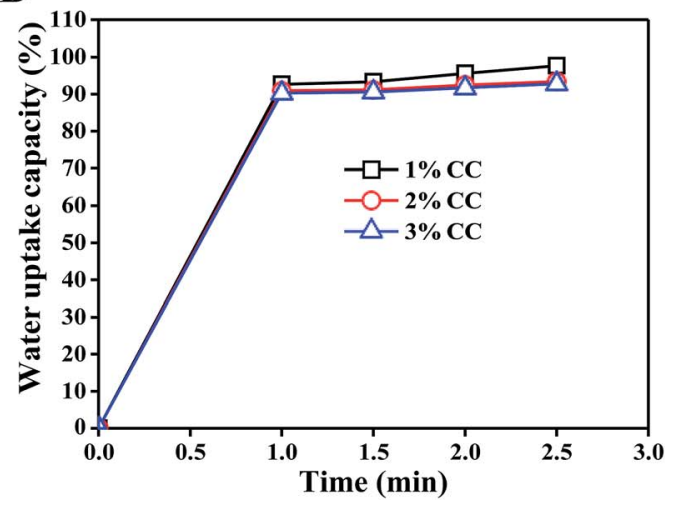

Fig. 3 (A) Swelling behavior and (B) water absorption capacity of CC hydrogels ( $1 \mathrm{wt} \%, 2 \mathrm{wt} \%$, and $3 \mathrm{wt} \%) * * p<0.01, n=3$. 

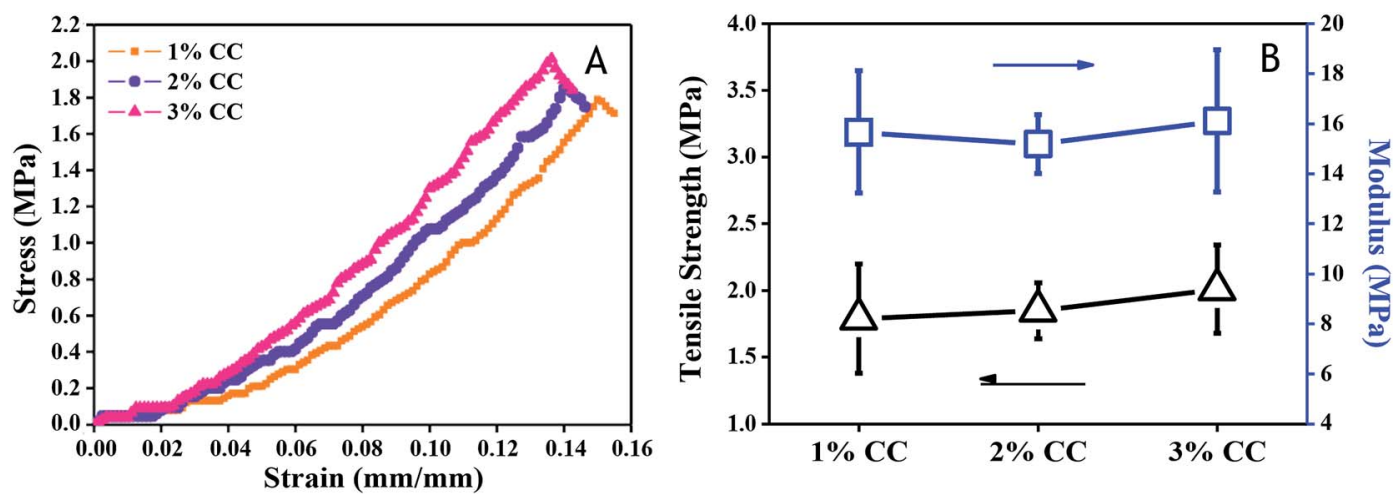

Fig. 4 Mechanical properties of 1 wt $\%, 2$ wt\%, and 3 wt\% CC hydrogels: (A) stress-strain behavior (B) tensile strength and modulus.

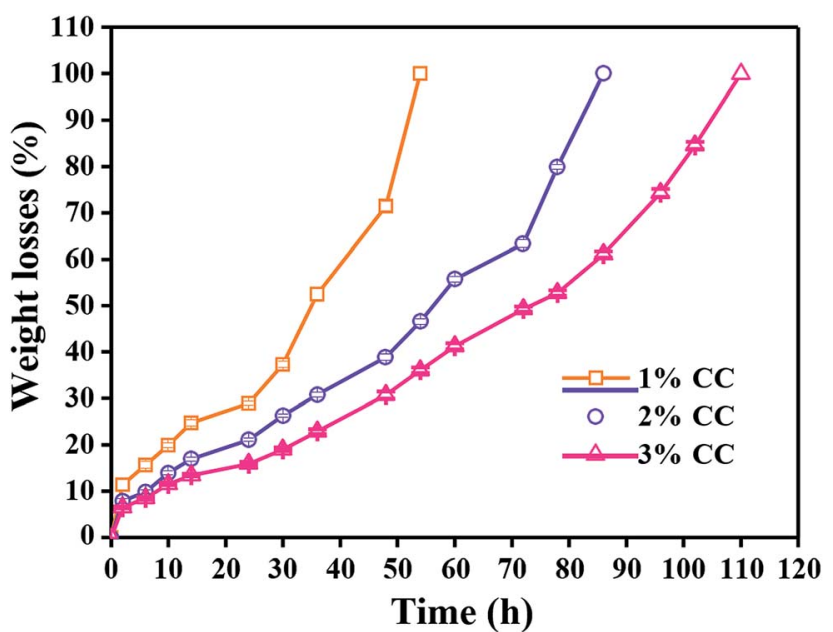

Fig. 5 In vitro degradation of $1 \mathrm{wt} \%, 2 \mathrm{wt} \%$ and $3 \mathrm{wt} \% \mathrm{CC}$ hydrogels by lysozyme as a function of incubation time $(n=5)$.

time for complete degradation of CC hydrogels, which are practical for wound care because the longer degradation time results in a longer time for wound coverage with the gel.

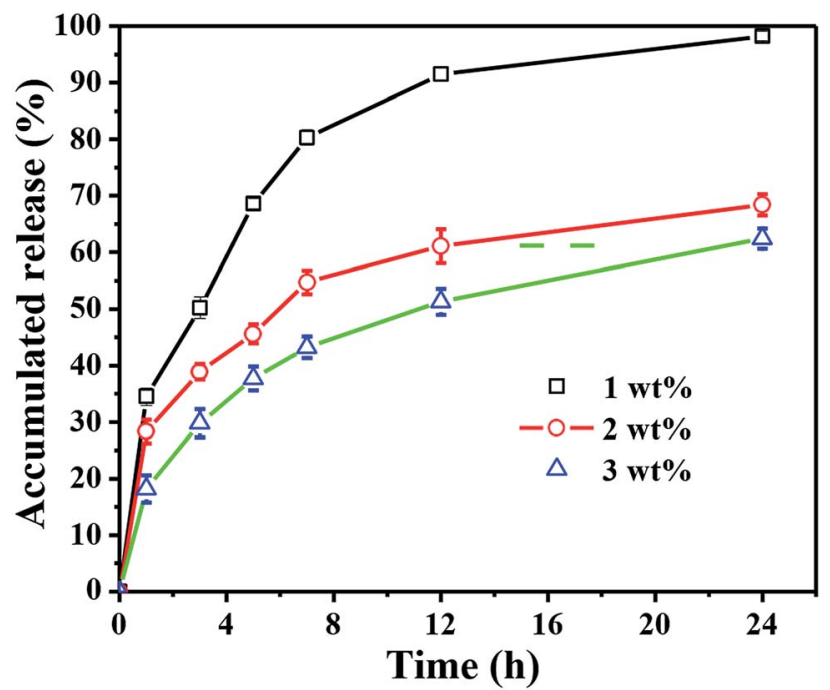

Fig. 6 Drug release behaviour of $1 \mathrm{wt} \%, 2 \mathrm{wt} \%$ and $3 \mathrm{wt} \% \mathrm{CC}$ hydrogels with release time.

\subsection{Drug loading and release}

To An ideal antimicrobial wound dressing should maintain a long period of drug controlled release to avoid the frequent changing of the dressing for reducing the risk of over exposing the patients to excess antimicrobial agents. ${ }^{36}$ The incorporation of $\mathrm{TH}$ as the model drug into the CC hydrogel was monitored for the release behaviour (Fig. 6). The cumulative release of $\mathrm{TH}$ from $1 \mathrm{wt} \%$ CC hydrogel exhibited an obvious burst release of $91.5 \pm 1.3 \%$ within $12 \mathrm{~h}$, which was significantly higher than $2 \mathrm{wt} \%$ and $3 \mathrm{wt} \% \mathrm{CC}$ hydrogel. After $24 \mathrm{~h}$, the cumulative release of $1 \mathrm{wt} \%$ CC hydrogel reached to a value of $98.2 \pm 0.9 \%$. The reason why the cumulative release of $\mathrm{TH}$ from 1 wt\% CC hydrogel was higher is that $1 \mathrm{wt} \%$ CC hydrogel posed the highest porosity and swelling ratios. Compared with $1 \mathrm{wt} \% \mathrm{CC}$ hydrogels, $2 \mathrm{wt} \%$ and $3 \mathrm{wt} \%$ CC hydrogels had better sustained release effect over an initial period of $7 \mathrm{~h}$. The cumulative release of TH from $2 \mathrm{wt} \%$ CC hydrogel gradually came to about $68.4 \pm 1.9 \%$ after $24 \mathrm{~h}$. As for the $3 \mathrm{wt} \% \mathrm{CC}$ hydrogel, the cumulative release increased gradually to $62.4 \pm 1.8 \%$ after

CC

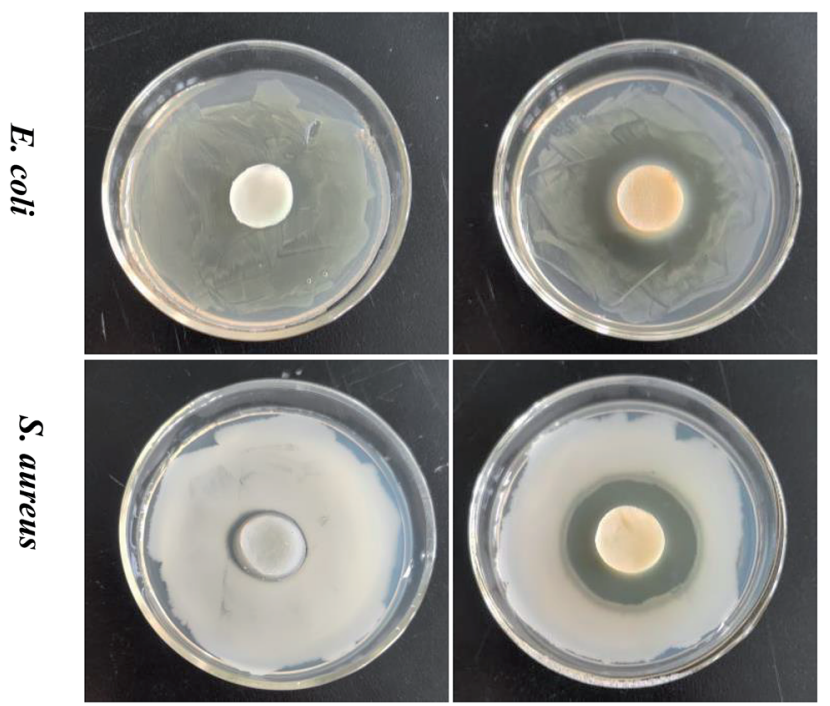

Fig. 7 Zone of inhibition studies of CC hydrogels against $E$. coli and S. aureus with/without drug. 
$24 \mathrm{~h}$, which was significantly steady by contrast with the release value of $2 \mathrm{wt} \% \mathrm{CC}$ hydrogel. These results demonstrated that the $3 \mathrm{wt} \%$ CC hydrogel is capable of drug controlled delivery with good properties of delayed release.

\subsection{Evaluation of the antimicrobial activity of TH-CC hydrogels}

Wound infection has been shown to impair wound contraction in both acute and chronic wounds, through the release of bacterial enzymes and metalloproteinases, which may degrade fibrin as well as wound growth factors. The fibrin matrix, especially fibronectin, is required for fibroblast migration, and it is also necessary for the maintenance of macrophage phagocytic activity. ${ }^{37}$ Taking this into account it is very important to develop wound dressings that also have bactericidal activity. The results obtained for the evaluation of the antimicrobial activity (Fig. 7). However, limited zone of inhibition was observed around CC hydrogel for both E. coli and S. aureus, and was completely surrounded by bacterial colonies. There was no microbial growth on the CC hydrogels surface indicating that $\mathrm{CC}$ acts as the contact killer for the microbes. Once drug is loaded onto the CC hydrogels, the drug is released into the surrounding medium and plays inhibitory role by killing the microbes.

\subsection{Treatment of wounds with TH-CC hydrogels}

Based on the above results, the efficacy of TH-CC hydrogels in repairing full-thickness skin wounds in rats was evaluated, compared with CC hydrogel and the dry cotton gauze (for dry healing). Images of the full layer wounds treated with dry cotton gauze, CC and TH-CC hydrogels are shown in Fig. 8A. The wound areas in all the three groups became smaller with increasing postsurgery time. After treated for $4 \mathrm{~d}$, the wound areas treated with TH-CC hydrogels were smaller than dry cotton gauze $(P<0.01)$. TH-CC hydrogels group also showed significant difference $(P<0.05)$ with CC hydrogels. However,

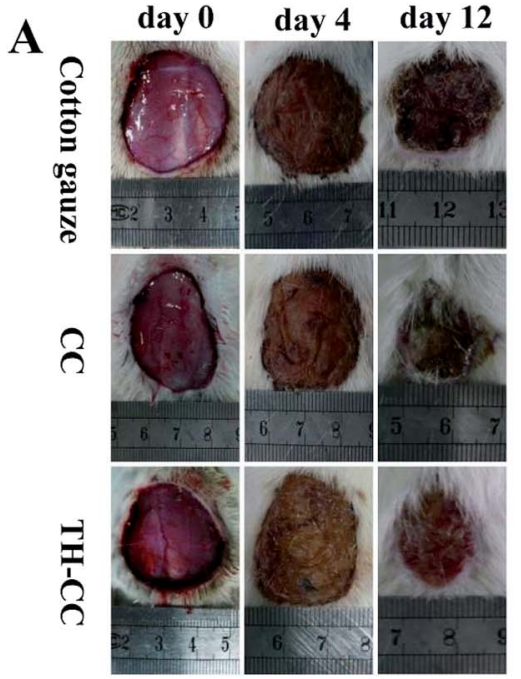

C
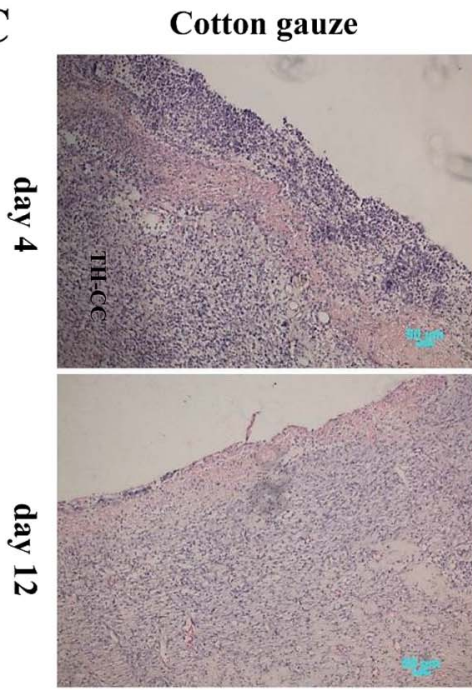

B

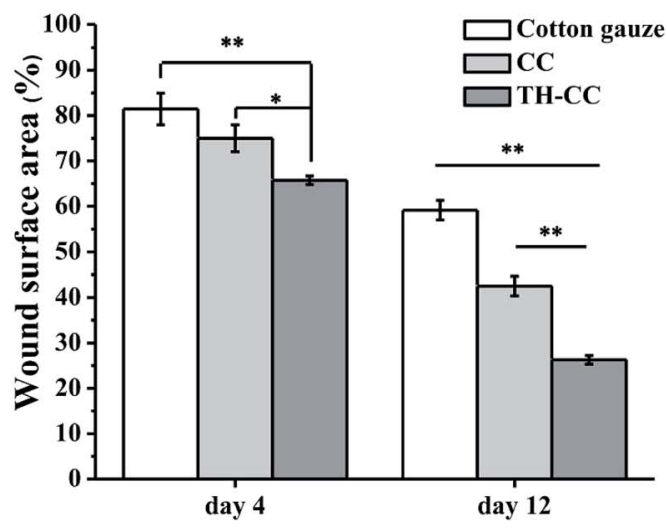

CC
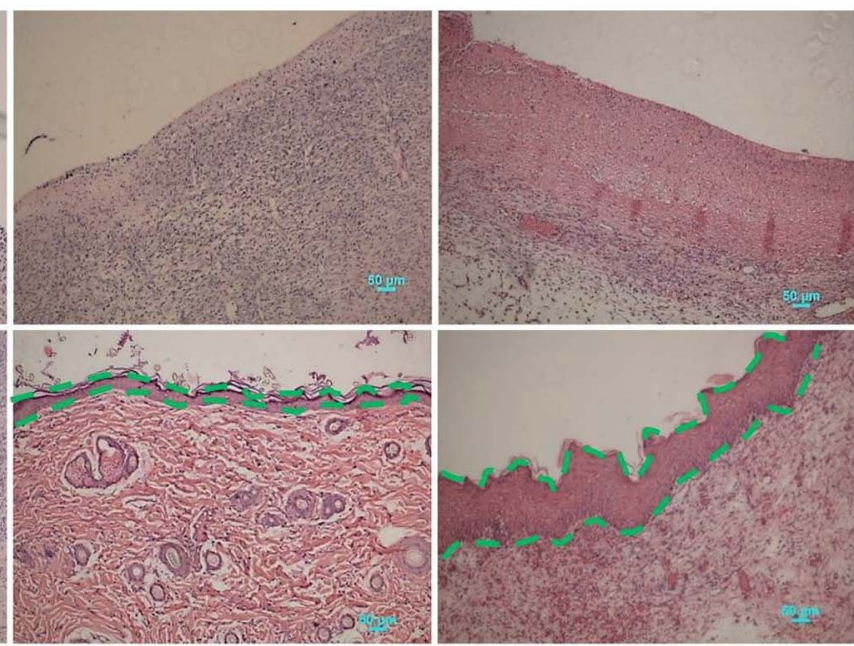

Fig. 8 (A) Photographs of the wound closure of rats covered with dry cotton gauze, CC and TH-CC hydrogel after $0,4,12$ days, respectively. (B) Wound area for each group. $* P<0.05, * * P<0.01,(n=6)$. (C) Hematoxylin- and eosin-stained sections of wound tissues treated with dry cotton gauze, $\mathrm{CC}$ and $\mathrm{TH}-\mathrm{CC}$ hydrogel after 4 days and 12 days postwounding (green lines: boundary of epithelium). 
there was no significant difference between CC hydrogel and dry cotton gauze groups $(P>0.05)$. After treated for $12 \mathrm{~d}$, TH-CC hydrogels showed significantly enhanced wound repair rate than CC hydrogels and dry cotton gauze group $(P<0.01)$. All these results demonstrated that the TH-CC hydrogels with antibacterial property showed great promoting effect in woundhealing compared with CC hydrogel and dry cotton gauze (Fig. 8B).

Fig. 8C shows the $\mathrm{HE}$ staining rat skin specimens of the wound covered with dry cotton gauze, CC and TH-CC hydrogel. The dry cotton gauze group had significantly largest numbers of inflammatory cells among three groups after 4 days. Although some inflammatory cells gathered between the epithelium and the muscle layer for the TH-CC hydrogel dressing, there was clear epidermal outward growth near the edge of the wound. The rate of epithelial cell migration to the wound site indicated that the wound repair efficacy was excellent. These results demonstrated that the TH-CC hydrogels did not cause increased foreign body response. After 12 days, prominent granulation tissue was observed in the treatments of the wounds covered with CC and TH-CC hydrogel, where the inflammatory cells almost disappeared. Meanwhile, granulation tissue and many blood vessels had formed in the area close to the surface of the lesion as well as underneath the newly growing epithelia. However, in the skin using a covering of dry cotton gauze covering, some inflammatory cells gathered around the wound area, indicating that the lesion was still in the period of early tissue remodeling. Between the hydrogel groups, TH-CC hydrogel produced a thicker epithelium tissue than CC hydrogel, indicating the best wound healing effect in these three groups.

\section{Conclusions}

The present study successfully demonstrated the applicability of the drug loaded CC hydrogels as a highly effective wound dressing with antibacterial efficacy. The proper analysis of the structure and the properties of these CC hydrogels have been systematically evaluated by SEM, swelling ratios and water absorption velocity, tensile strength, and in vitro degradation studies. The composite dressings exhibited excellent antibacterial activity against both $E$. coli and $S$. aureus, which is fundamental to avoid wound infection. A sustained drug release pattern was observed for the CC dressings. TH-CC dressings showed better wound healing effect than dry cotton gauze and CC hydrogel in a full-thickness mouse skin wound model. The presence of $\mathrm{TH}$ helps in protection from bacterial invasions. From these results, it is concluded that the TH-CC gels have potential application for use in wound care.

\section{Conflicts of interest}

There are no conflicts to declare.

\section{Acknowledgements}

This work was partially supported by the National Natural Science Foundation of China (31830028, 81801856 and 81370117), the Natural Science Foundation of Jiangsu Province (BK20180949), Six Talent Peaks Project in Jiangsu Province (XCL-063), Nantong applied basic research program (MS12017023-2), High Level Introduction of Talent Research Start-up Foundation of Nantong University (03081136, 03081135) and Large Instruments Open Foundation of Nantong University. We also would like to thank Analysis and Test Center of Nantong University for the testing support.

\section{Notes and references}

1 I. Y. Kim, S. J. Seo, H. S. Moon, M. K. Yoo, I. Y. Park, B. C. Kim and C. S. Cho, Biotechnol. Adv., 2008, 26, 1-21.

2 H. Hamedi, S. Moradi, S. M. Hudson and A. E. Tonelli, Carbohydr. Polym., 2018, 199, 445-460.

3 L. Zhou, Y. W. Xi, Y. M. Xue, M. Wang, Y. L. Liu, Y. Guo and B. Lei, Adv. Funct. Mater., 2019, 1806883, 1-11.

4 H. Chen, R. Y. Cheng, X. Zhao, Y. H. Zhang, A. Tam, Y. F. Yan, H. K. Shen, Y. S. Zhang, J. Qi, Y. H. Feng, L. Liu, G. Q. Pan, W. G. Cui and L. F. Deng, NPG Asia Mater., 2019, 11, 3 .

5 J. Qu, X. Zhao, Y. P. Liang, Y. M. Xu, P. X. Ma and B. L. Guo, Chem. Eng. J., 2019, 362, 548-560.

6 M. M. Chen, J. Tian, Y. Liu, H. Cao, R. Y. Li, J. H. Wang, J. L. Wu and Q. Q. Zhang, Chem. Eng. J., 2019, 373, 413-424.

7 Y. P. Liang, X. Zhao, T. L. Hu, B. J. Chen, Z. H. Yin, P. X. Ma and B. L. Guo, Small, 2019, 15, 1900046.

8 Z. Di, Z. Shi, M. W. Ullah, S. Li and G. Yang, Int. J. Biol. Macromol., 2017, 105, 638-644.

9 E. A. Kamoun, X. Chen, M. S. M. Eldin and E.-R. S. Kenawy, Arabian J. Chem., 2015, 8, 1-14.

10 J. Koehler, L. Verheyen, S. Hedtrich, F. P. Brandl and A. M. Goepferich, J. Biomed. Mater. Res., Part A, 2017, 105, 3360-3368.

11 F. Oeri, R. Dietrich, C. Ganz, M. Dau, D. Wolter, A. Kasten, T. Gerber and B. Frerich, J. Craniomaxillofac. Surg., 2017, 45, 99-107.

12 K. Murakami, H. Aoki, S. Nakamura, S.-i. Nakamura, M. Takikawa, M. Hanzawa, S. Kishimoto, H. Hattori, Y. Tanaka, T. Kiyosawa, Y. Sato and M. Ishihara, Biomaterials, 2010, 31, 83-90.

13 S. Anjum, A. Arora, M. S. Alam and B. Gupta, Int. J. Pharm., 2016, 508, 92-101.

14 T. Takei, H. Nakahara, H. Ijima and K. Kawakami, Acta Biomater., 2012, 8, 686-693.

15 Y. C. Chung, H. L. Wang, Y. M. Chen and S. L. Li, Bioresour. Technol., 2003, 88, 179-184.

16 D. Zhang, W. Zhou, B. Wei, X. Wang, R. Tang, J. Nie and J. Wang, Carbohydr. Polym., 2015, 125, 189-199.

17 I. R. Sweeney, M. Miraftab and G. Collyer, Carbohydr. Polym., 2014, 102, 920-927.

18 S. Sakai, M. Khanmohammadi, A. B. Khoshfetrat and M. Taya, Carbohydr. Polym., 2014, 111, 404-409. 
19 E. S. Costa-Junior, E. F. Barbosa-Stancioli, A. A. P. Mansur, W. L. Vasconcelos and H. S. Mansur, Carbohydr. Polym., 2009, 76, 472-481.

20 H. S. Mansur, E. d. S. Costa Jr, A. A. P. Mansur and E. F. Barbosa-Stancioli, Mater. Sci. Eng., C, 2009, 29, 15741583.

21 V. S. Kumari, S. K. Basha and P. N. Sudha, Polym. Bull., 2012, 68, 1387-1393.

22 J. M. Yang, W. Y. Su, T. L. Leu and M. C. Yang, J. Membr. Sci., 2004, 236, 39-51.

23 A. Sanchez-Ferrero, A. Mata, M. A. Mateos-Timoneda, J. C. Rodriguez-Cabello, M. Alonso, J. Planell and E. Engel, Biomaterials, 2015, 68, 42-53.

24 P. Ghosh, A. P. Rameshbabu and S. Dhara, Langmuir, 2014, 30, 8442-8451.

25 H. Chen, H. Wang, B. Li, B. Feng, X. He, W. Fu, H. Yuan and Z. Xu, RSC Adv., 2018, 8, 16910-16917.

26 S. Eswaranandam, N. S. Hettiarachchy and M. G. Johnson, J. Food Sci., 2004, 69, FMS79-FMS84.

27 S. Anjum, A. Gupta, D. Sharma, D. Gautam, S. Bhan, A. Sharma, A. Kapil and B. Gupta, Mater. Sci. Eng., C, 2016, 64, 157-166.
28 N. Kathuria, A. Tripathi, K. K. Kar and A. Kumar, Acta Biomater., 2009, 5, 406-418.

29 S. H. Chen, C. T. Tsao, C. H. Chang, Y. T. Lai, M. F. Wu, C. N. Chuang, H. C. Chou, C. K. Wang and K. H. Hsieh, Mater. Sci. Eng., C, 2013, 33, 2584-2594.

30 S. Tanodekaew, M. Prasitsilp, S. Swasdison, B. Thavornyutikarn, T. Pothsree and R. Pateepasen, Biomaterials, 2004, 25, 1453-1460.

31 E. Lih, J. S. Lee, K. M. Park and K. D. Park, Acta Biomater., 2012, 8, 3261-3269.

32 C. Jacquemoud, K. Bruyere-Garnier and M. Coret, J. Biomech., 2007, 40, 468-475.

33 F. H. Silver, J. W. Freeman and D. DeVore, Skin Res. Technol., 2001, 7, 18-23.

34 J. Manschot and A. Brakkee, J. Biomech., 1986, 19, 511-515.

35 D. W. Ren, H. F. Yi, W. Wang and X. J. Ma, Carbohydr. Res., 2005, 340, 2403-2410.

36 J. S. Boateng, K. H. Matthews, H. N. E. Stevens and G. M. Eccleston, J. Pharm. Sci., 2008, 97, 2892-2923.

37 W. K. Stadelmann, A. G. Digenis and G. R. Tobin, Am. J. Surg., 1998, 176, 39S-47S. 